\title{
Editorial: An Introduction to the Special Section on Organizational Behavior Management in Health \& Human Services
}

\author{
Manuel A. Rodriguez ${ }^{1,2} \cdot$ Heather M. McGee ${ }^{3}$ \\ Published online: 26 November 2019 \\ (C) Association for Behavior Analysis International 2019
}

In 2017, the Association for Behavior Analysis International conference held a special one-day pre-conference on Organizational Behavior Management (OBM) in Health and Human Services (HHS). This pre-conference featured experts presenting on topics that highlight the breadth and range of OBM, particularly as it is applied within HHS. Dr. Aubrey Daniels kicked off the day's events with an introduction to OBM told through the lens of his 40 years of experience and expertise as a leading OBM consultant and author.

While some of the talks presented behavioral systems analysis (BSA) and largescale change, others targeted performance management (PM) and day-to-day supervision and management requirements, demonstrating that OBM is useful for both strategic and tactical organizational performance improvement. Presenting on BSA and large-scale change, Dr. Heather McGee kicked things off with her talk on how HHS organizations can use BSA to implement large-scale change initiatives. Brett DiNovi presented on the specific leadership accomplishments and behaviors required to successfully and sustainably grow organizations serving individuals with ASD. Dr. Mark Dixon described system-wide interventions that OBMers and HHS organizational leaders could use to promote both sound financial and clinical practices. Drilling down into the PM side of OBM, Dr. Donald Hantula described how OBM can and should be incorporated into supervision practices to ethically improve and support employee performance, while Dr. Florence DiGennaro Reed presented a three-term model for ongoing staff training and performance management. Finally, Dr. Nicholas Weatherly presented an OBM approach to building a clinical safety culture to improve employee safety performance.

Manuel A. Rodriguez

manny@buenoventures.com

1 Bueno Ventures Management Services, Inc., Doral, FL, USA

2 MUMA College of Business, University of South Florida, Tampa, FL, USA

3 Western Michigan University, Kalamazoo, MI, USA 
Wrapping up the day's presentations, Dr. Robyn Catagnus, Dr. Jane Howard, Rita Gardner, and Dr. Linda LeBlanc participated a panel discussion on the executive perspective in HHS organizations. They used their first-hand experience as leaders in these organizations to provide real-world examples of HHS organizations that have embraced an OBM approach. Building on the panel discussion, the pre-conference concluded with an organizational showcase, where leaders of various human service organizations met with small groups of attendees to discuss applications of OBM within their organizations.

A lot of great information was presented during the ABAI 2017 one-day, preconference on OBM in HHS. This special issue of Perspectives on Behavior Science provides a representative selection of those presentations. We are pleased to provide this special issue and hope the $P o B S$ readership finds the papers as enlightening as the attendees found the talks at the conference. The special edition begins with McGee and Crowley-Koch's (this issue) walk-through of how BSA can be used to build a comprehensive strategic and tactical plan for a large scale initiative within an autism service provider. The authors provide a brief history of BSA, followed by a series of systemsbased questions that should be asked and answered before engaging in a large-scale change initiative. The authors describe the risks of taking a "quick" approach versus a more "planned approach" to change, but wrap up their paper with a BSA approach to change analysis for organizations that have already taken the "quick" approach.

The next article in this special issue focuses on the day-to-day management of employee performance within human service organizations. Novak, DiGennaro Reed, Erath, Blackman, Ruby, and Pellegrino (this issue) introduce the behavioral skills training (BST) paradigm (instruction, modeling, rehearsal, feedback) and provide practice guidelines for the reader. The authors also provide considerations for a supervision path for ongoing staff support. This path goes beyond BST for new supervisees/employees to describe the supervisor's responsibilities around conducting employee observations, delivering effective performance feedback, incorporating pay for performance systems, assessing performance problems, and identifying and providing opportunities for continuing education. This article highlights the important role performance management plays in effective service delivery, and the socially significant impact OBM can have on staff and consumers of health and human services.

The first article in this special issue provides readers with an example of how OBM can be used to broadly improve organizational systems through BSA. The second article provides readers with an example of how OBM can be used to improve frontline employee performance through BST and PM. In the final article based on the preconference talks, Weatherly (this issue) describes how OBM can be used to improve a specific aspect of employee performance - safety. Weatherly presents a clear rationale for building a safety culture in clinical service settings, what such a culture comprises, from a behavioral perspective, and the role of leadership in the design and implementation of such a system. He provides readers with a behavioral safety model that can be applied to any health and human services organization. The model includes safety metrics, assessment of variables influencing safety, and assessment-based solutions. Additionally, Weatherly dedicates a portion of the article to the development of coaching systems to positively influence safety across the organizational culture.

This special issue concludes with a book review. While this review is not the product of a talk given at the ABAI 2017 OBM in HHS pre-conference, the publication of 
Pritchard and Wine's (2018) book, Organizational Behavior Management: The Essentials, between the conference and the publication of this special issue was too happy a coincidence to pass up! Our sincerest thanks to Dr. Sharlet Rafacz for providing this timely book review, which is unique in that it is written from the perspective of an OBM course instructor, who is the likely audience for such a book. Rafacz (this issue) has built her review and recommendations around specific chapters in terms of their appropriateness for either an undergraduate or graduate level course (or both). The review provides a summary of the various sections and chapters of the book as well, with points of reflection on strengths and weaknesses. The book review concludes with a perspective on the utility of the book as a whole, the contributions it offers to our field, and considerations for future editions.

Finally, we would be remiss not to remind the readers that there is much more to OBM in health and human services - and OBM in general - than what we have provided in this special issue. The field of applied behavior analysis has a long, successful history of producing therapeutic gains within the human services industry, and OBM has been an integral piece of this success. In fact, Sturmey (2010) described OBM and ABA in developmental disabilities as "old friends," or as Ludwig (2015) notes, OBM is the enabler of ABA. OBM has implemented successfully in many types of health and human service settings, including hospital emergency rooms (Kelley \& Gravina, 2018), outpatient medical clinics (Slowiak, 2014), physical therapy clinics (Gravina, VanWagner, \& Austin, 2008), brain injury treatment centers (Guercio \& Dixon, 2011), schools (Gavoni and Rodriguez, 2016; Phillips, 1998), special education (Maher, 1981), residential treatment centers (Gil and Carter, 2016; Parsons, Cash \& Reed, 1989; Sasaki and Noro, 2017), psychiatric group homes (Huberman \& O’Brien, 1999) and therapeutic and educational organizations that serve individuals with autism (Cook and Dixon, 2005; Miller, Carlson, Sigurdsson, 2014). In fact, the first issue of the Journal of Organizational Behavior Management (JOBM), published in 1977, included studies conducted in human service settings that aimed to reduce absenteeism, implement token economies, and assess the impact of feedback on mental health technician performance. More recently, applications of OBM can be seen in studies on improving staff training (Shapiro and Kazemi, 2017), use of technology on data collection (Sleeper, LeBlanc, Mueller, and Valentino, 2017), and even in a compilation of case studies by Reid, Parsons, and Jensen (2018) related to improving staff performance. In their recent literature review, Gravina, Villacorta, Albert, Clark, Curry, and Wilder (2017) demonstrated the breadth and depth of OBM's successful implementation within HHS organizations. We highly encourage readers interested in OBM to seek out the wealth of literature that is available on this topic.

\section{References}

Cook, T., \& Dixon, M. R. (2005). Performance feedback and probabilistic bonus contingencies among employees in a human service organization. Journal of Organizational Behavior Management, 25(3), 45-63.

Gavoni, P., \& Rodriguez, M. (2016). Quick Wins! Accelerating School Transformation through Science, Engagement, and Leadership. Melbourne, FL: USA: ABA Technologies, Inc. First Edition. 
Gil, P. J., \& Carter, S. L. (2016). Graphic feedback, performance feedback, and goal setting increased staff compliance with a data collection task at a large residential facility. Journal of Organizational Behavior Management, 36(1), 56-70. https://doi.org/10.1080/01608061.2016.115220.

Gravina, N., VanWagner, M., \& Austin, J. (2008). Increasing physical therapy equipment preparation using task clarification, feedback and environmental manipulations. Journal of Organizational Behavior Management, 28(2), 110-122 https://doi-org.libproxy.temple.edu/10.1080/01608060802100931.

Gravina, N., Villacorta, J., Albert, K., Clark, R., Curry, S., \& Wilder, D. (2017). A Literature Review of Organizational Behavior Management Interventions in Human Service Settings from 1990 to 2016. Journal of Organizational Behavior Management 2018, 38(2-3), 191-224. https://doi.org/10.1080 /01608061.2018.1454872.

Guercio, J. M., \& Dixon, M. R. (2011). The observer effect and its impact on staff behavior in an acquired brain injury neurobehavioral treatment setting. Journal of Organizational Behavior Management, 31(1), 43-54 https://doi-org.libproxy.temple.edu/10.1080/01608061.2010.520142.

Huberman, W. L., \& O'Brien, R. M. (1999). Improving therapist and patient performance in chronic psychiatric group homes through goal-setting, feedback, and positive reinforcement. Journal of Organizational Behavior Management, 19(1), 13-36 https://doi-org.libproxy.temple.edu/10.1300/J075 v19n01_04.

Kelley III, D. P., \& Gravina, N. (2018). Every minute counts: Using process improvement and performance feedback to improve patient flow in an emergency department. Journal of Organizational Behavior Management, 38(2-3), 234-243 https://doi-org.libproxy.temple.edu/10.1080/01608061.2017.1423150.

Ludwig, T. D. (2015). Organizational behavior management: An enabler of applied behavior analysis. In H. S. Roane, J. E. Ringdahl, \& T. S. Falcomata (Eds.), Clinical and organizational applications of applied behavior analysis (pp. 605-625). San Diego, CA: Elsevier Academic Press https://doi-org.libproxy. temple.edu/10.1016/B978-0-12-420249-8.00024-1.

Maher, C. A. (1981). Improving the delivery of special education and related services in public schools. Journal of Organizational Behavior Management, 3(1), 29-44.

Miller, M. V., Carlson, J., \& Sigurdsson, S. O. (2014). Improving treatment integrity in a human service setting using lottery-based incentives. Journal of Organizational Behavior Management, 34(1), 29-38.

Parsons, M. B., Cash, V. B., \& Reed, D. H. (1989). Improving residential treatment services: Implementation and norm-referenced evaluation of a comprehensive management system. Journal of Applied Behavior Analysis, 22, 143-156.

Phillips, J. R. (1998). Applications and contributions of organizational behavior management in schools and day treatment settings. Journal of Organizational Behavior Management, 18(1-2), 103-129.

Reid, P., \& Jensen. (2018). Maintaining Staff Performance Following a Training Intervention: Suggestions from a 30-Year Case Example. Behavior Analysis in Practice, 10, 12-21.

Saski, G., \& Noro, F. (2017). A Plan, Do, Check, Act Cycle of Support for Children in Residential Care: A Staff Management Intervention. Journal of Special Education Research, 6(1), 11-23.

Shapiro and Kazemi. (2017). A Review of Training Strategies to Teach Individuals Implementation of Behavioral Interventions. Journal of Organizational Behavior Management, 37(1), 32-62.

Sleeper, L. B., Mueller, \& Valentino. (2017). The Effects of Electronic Data Collection on the Percentage of Current Clinician Graphs and Organizational Return on Investment. Journal of Organizational Behavior Management, 37(1), 83-95.

Slowiak, J. M. (2014). "how may I help you?" improving telephone customer service in a medical clinic setting. Journal of Organizational Behavior Management, 34(1), 39-51 https://doi-org.libproxy.temple. edu/10.1080/01608061.2013.873382.

Publisher's Note Springer Nature remains neutral with regard to jurisdictional claims in published maps and institutional affiliations. 